\title{
Focal cartilage defects of the lateral compartment do influence the outcome after high tibial valgus osteotomy
}

\author{
Tizian Heinz ${ }^{1}$, Stephan Reppenhagen ${ }^{1}$, Mike Wagenbrenner ${ }^{1}$, Konstantin Horas ${ }^{1}$, Malte Ohlmeier ${ }^{2}$, \\ Thomas Schäfer $^{1}$, Maximilian Rudert ${ }^{1}$, Thomas Barthel ${ }^{1}$, and Manuel Weißenberger ${ }^{1, *}$ \\ ${ }^{1}$ Department of Orthopaedic Surgery, University of Wuerzburg, Koenig-Ludwig-Haus, Brettreichstr. 11, 97074 Wuerzburg, Germany \\ 2 Department of Orthopaedic Surgery, HELIOS ENDO-Klinik Hamburg, 22767 Hamburg, Germany
}

Received 29 June 2021, Accepted 27 July 2021, Published online 25 August 2021

\begin{abstract}
Introduction: High tibial medial open-wedge valgus osteotomy (HTO) is a well-established procedure for unicompartimental medial osteoarthritis of the young and active patient. However, the influence of cartilage defects of the lateral compartment on the total outcome remains obscure. Methods: From 2005 to 2012, a total of 63 patients underwent HTO for medial osteoarthritis of the knee at a single university orthopaedic center. Baseline data as well as intraoperative findings, including the grade and location of cartilage lesions, were evaluated retrospectively. Two groups were formed regarding the integrity of the lateral tibiofemoral compartment as measured by the Outerbridge score (group A: no lateral cartilage defects, group B: mild to moderate lateral cartilage defects). Functional outcome was assessed using the Knee and Osteoarthritis Outcome Score (KOOS), including its five subscores. Results: Comparing pre- and postoperative data, we identified an overall benefit of the HTO procedure as measured by the KOOS. Group A (no lateral cartilage defects) showed an increase in all five KOOS subscores $(p=0.00-0.01$ ), whereas for group B (mild to moderate lateral cartilage defects), only two KOOS subscores revealed a significant increase $(p=0.03-0.04)$. There was also a statistically significant difference in the total KOOS score with higher values for group A at the postoperative visit. Cartilage defects with a higher Outerbridge score were associated with lower postoperative KOOS subscores. Discussion: Mild to moderate cartilage defects of the lateral compartment humble the total outcome after HTO procedure. Thus, indication for HTO should be made very carefully if any degree of lateral cartilage degeneration is present.
\end{abstract}

Key words: Knee, Medial osteoarthritis, High tibial osteotomy, Cartilage defect.

\section{Introduction}

Osteoarthritis (OA) is one of the most common musculoskeletal disorders, especially among the elderly population. Optimal treatment strategies, especially for younger patients, are scarce. Moreover, the available strategies are controversially discussed in current literature. In Germany, the total 12-months prevalence of osteoarthritis has been reported to be $17.9 \%$ of adults over 18 years. A steep increase by age was recognized, reaching as high as $48.1 \%$ of affected women aged over 65 years [1]. Risk factors for osteoarthritis can be divided into non-modifiable and modifiable factors, with malalignment of the lower extremity being assigned to the latter. Various studies have demonstrated that malalignment can cause increased stress across a focal joint area and thus leading to subsequent cartilage loss and osteoarthritis [2-4]. Even in a neutrally aligned limb, the force distribution across the knee joint is not completely equally distributed between the medial and lateral compartment.
In particular, 60-70\% of the force is directed to the medial compartment during weight-bearing activities. This may explain the higher prevalence of the medially emphasized osteoarthritis of the knee compared to lateral osteoarthritis [5-9]. In a varus aligned knee, the load-bearing mechanical axis is shifted to the medial compartment leading to a 7.4-fold increased body weight force across this joint area during walking and a fourfold increased risk of progression of medial OA [10, 11]. While there is a broad consensus for the use of total knee arthroplasty (TKA) in late-stage, symptomatic bicompartmental knee OA amongst orthopaedic surgeons, operative treatment strategies for unicompartmental medial OA remain controversial. Both unicompartmental knee arthroplasty (UCA) and high tibial valgus osteotomy (HTO) are recognized and well-accepted treatment options for medial OA of the knee [12-15]. However, the right and thoughtful patient assignment to the aforementioned procedures represents a critical step to gain favorable and satisfactory outcome parameters. This study aimed to

\footnotetext{
*Corresponding author: m-weissenberger.klh@uni-wuerzburg.de
} 
assess the functional outcome as measured by the Knee and Osteoarthritis Outcome Score (KOOS) after HTO for osteoarthritis of the medial compartment in varus aligned knees was performed. Special interest was put on how cartilage defects of the lateral compartment impact the total postoperative outcome score. We hypothesized that even with mild to moderate cartilage defects of the lateral compartment expectations on HTO should be humbled.

\section{Materials and methods}

\section{Patient recruitment and study population}

A retrospective study of patients undergoing HTO due to varus deformity of the proximal tibia was performed. Data analysis was performed using an electronic medical report system. From August 2005 to March 2012, a total of 63 patients were included in this study. Inclusion criteria were an ongoing complaint of knee pain, osteoarthritis of the medial compartment with a Kellgren-Lawrence-Score of not more than three or a focal cartilage lesion of the medial compartment, patient age under 60 years, and knee flexion of at least $120^{\circ}$. Highgrade ligamentous instabilities, as defined by simultaneously present coronal and sagittal laxity of the knee, as well as inflammatory arthropathy, were defined as exclusion criteria. Furthermore, mild to moderate patellar lesions (Outerbridge $\geq 2$ ) lead to exclusion from the study. In every case, a weight-bearing anteroposterior long-axis view was obtained before surgery to determine the preoperative knee alignment. Calculation of the planned correction osteotomy was made electronically using planning software (mediCAD, Version 3.0, Hectec GmbH, Germany). Six weeks postoperatively, another weight-bearing anteroposterior long-axis view was obtained. From 2005 to 2012, a total of 63 patients underwent HTO by meeting the aforementioned criteria in a single orthopaedic center. In every case, a medial open-wedge approach was used and a Tomofix ${ }^{\circledR}$ plate secured the osteotomy. Written informed consent for participation was obtained from every individual and the study had been approved by the ethics committee of The University of Wuerzburg.

\section{Surgical treatment and postoperative rehabilitation}

Diagnostic arthroscopy of the knee joint was routinely performed before HTO to evaluate the current state of cartilage degeneration of the medial and lateral compartment. Further, the degree of meniscal degeneration and ligamentous injuries were assessed. Meniscal injuries detected during diagnostic arthroscopy were addressed via a partial resection of the injured pattern. The same applied to areas of degenerated cartilage which were addressed via debridement of the affected area. Cartilage lesions were classified according to the Outerbridge classification system. Both for the medial and lateral compartment, a total Outerbridge score was calculated by summarizing the highest cartilage defect of the femoral condyle and the corresponding tibial plateau of one compartment. Thus, a score ranging from 0 to 8 could be formed for each compartment, with a score of 8 indicating a progressive cartilage degeneration of one compartment. Therefore, the authors considered a score of one to three as a mild cartilage degeneration. Accordingly, score values between four and six were considered as moderate cartilage defects of the medial or lateral compartment. Score values higher than six were defined as progressive cartilage defects. For better comparability, two groups were formed afterwards: group A with no cartilage defects (total lateral Outerbridge score 0 ) and group B with mild to moderate cartilage degeneration of the lateral compartment (total lateral Outerbridge score one to six). After the diagnostic arthroscopy, the HTO procedure followed subsequently. Therefore, the medial proximal tibia was exposed by a longitudinal incision. A biplanar osteotomy was then performed by an ascending cut starting from the medial border of the tibia towards the tibiofibular joint followed by a subsequent tuberosity cut parallel to the anterior tibial margin. The osteotomy was opened gradually using chisels and a spreading device. After the desired amount of tibial opening has been achieved, the osteotomy was secured by a Tomofix ${ }^{\circledR}$ plate. The aim of correction was defined as a slight valgus of $3-5^{\circ}$ relative to the mechanical axis defined by Dugdale et al. and thus unloading the medial compartment [16]. Postoperatively, all patients underwent a standardized rehabilitation protocol. Continuous passive motion without resistance was recommended from day one postoperatively for six weeks without weight-bearing of the treated extremity. Weight-bearing anteroposterior long-axis views were obtained six weeks postoperatively, and if the bone healing was considered sufficiently, a stepwise increase until full weight bearing from 9 to 10 weeks postoperatively was achieved.

\section{Follow-up evaluation}

The German version of the Knee and Osteoarthritis Outcome Score (KOOS) was used to assess the surgical outcome after HTO was performed. The KOOS has been validated for various pathologies of the knee, including OA [17]. The German version of the KOOS has also been validated for measuring therapeutic effects of knee-related conditions [18]. The KOOS questionnaires were handled out to the patients postally, and both the pre- and postoperative condition was evaluated retrospectively.

\section{Statistical analysis}

Data management and analysis were conducted using IBM SPSS Statistics version 26 (IBM Corp., Armonk, NY, USA). Baseline variables and the KOOS outcome score were assessed using standard descriptive statistics. For continuous variables, means and standard deviations were calculated, whereas absolute and relative frequencies are shown for categorial variables. Data were checked for normal distribution using the Kolmogorov-Smirnov test and Shapiro-Wilk test. $P$-values $<0.05$ were considered statistically significant. For normally distributed data, comparisons were made using Student's $T$-test. For not normally distributed data, the Mann-Whitney- $U$ test and Kruskal-Wallis test were performed. Correlations were identified based on the Pearson correlation coefficient, respectively, 
Table 1. Demographic and surgical data of Group A and Group B at the time of surgery.

\begin{tabular}{lccc}
\hline & $\begin{array}{c}\text { Group A (no lateral } \\
\text { cartilage lesions) }\end{array}$ & $\begin{array}{c}\text { Group B (mild to moderate } \\
\text { lateral cartilage lesions) }\end{array}$ & $\begin{array}{c}\text { P-value for statistical } \\
\text { difference }\end{array}$ \\
\hline Age (years) & $39.2 \pm 12.9$ & $45.2 \pm 7.9$ & 0.06 \\
BMI (kg/m) & $25.6 \pm 4.9$ & $27.9 \pm 4.3$ & 0.09 \\
Preoperative varus deformitiy (in ${ }^{\circ}$ ) & $7.7 \pm 2.3$ & $6.5 \pm 3.3$ & 0.17 \\
Kellgren-Lawrence medial compartment & $2.2 \pm 0.5$ & $2.6 \pm 0.7$ & $0.01^{*}$ \\
Kellgren-Lawrence lateral compartment & $1.3 \pm 0.5$ & $1.4 \pm 0.6$ & 0.49 \\
Ratio male : female patients & $34: 5$ & $20: 4$ & - \\
Outerbridge score lateral femoral condyle & - & $1.1 \pm 0.7$ & - \\
Outerbridge score lateral tibial plateau $_{\text {aMPTA, preoperative (in }{ }^{\circ} \text { ) }}$ & - & $1.4 \pm 0.6$ & - \\
aMPTA, postoperative (in $^{\circ}$ ) & $84.0 \pm 2.4$ & $84.4 \pm 3.1$ & 0.57 \\
\hline
\end{tabular}

Significant differences are highlighted by an asterisk. aMPTA: anatomical medial proximal tibial angle.

the Spearman's correlation coefficient for metrically and ordinally scaled variables.

\section{Results}

\section{Patient demographics}

In the whole study cohort, the mean patient age was 39 years (range from 14 to 58 years), and the mean body-massindex (BMI) was $27 \mathrm{~kg} / \mathrm{m}^{2}\left(17-40 \mathrm{~kg} / \mathrm{m}^{2}\right)$. The left and right extremity were nearly equally affected (51\% vs. $49 \%)$. Of the 63 patients included in the study, 54 were males and 9 were females. Details regarding demographic and preoperative findings, specifically of the two considered study groups, are depicted in Table 1. Fifteen patients (22\%) stated to have a regulatory intake of nicotine of at least three cigarettes per day. The mean Kellgren-Lawrence-Score was $2.3 \pm 0.6$ for the medial compartment and $1.3 \pm 0.5$ for the lateral compartment, respectively. The mean Outerbridge score for the medial compartment was significantly higher than the lateral compartment $(4.8 \pm 2.5$ vs. $2.5 \pm 1.2, p<0.00)$. There was a statistical difference in the Kellgren-Lawrence-Score of the medial compartment between both groups ( $p=0.01)$ (Table 1). Bivariate correlation analysis revealed a poor correlation of the Kellgren-Lawrence-Score of the medial compartment with the Outerbridge-Score of the lateral compartment $(r=0.33, p=0.01)$. The mean anatomical medial proximal tibia angle (aMPTA) before surgery was $84.2^{\circ} \pm 2.6$ (range 75.4-88.7) compared to $92.5^{\circ} \pm 3.3$ (range 84.9-98.9) in the postoperative radiographs. Preoperative values of the aMPTA below 88.0 were considered a pathological tibial varus deformity [19]. The mean follow-up period averaged $46.1 \pm 24.3$ months (10.0-93.0 months). The medial wedge was left unfilled or filled using autologous spongiosa or bone graft substitute (Table 2). Details on the mean Outerbridge score of the medial and lateral femorotibial compartment are given in Table 3 .

\section{Meniscal and ligamentous integrity}

Nearly one-third of the patients showed to have an intact medial meniscus at the time of the surgery. Seventy percent had either a stable partially resected medial meniscus due to
Table 2. The medial wedge was left unfilled or was reconstructed using bone substitute.

\begin{tabular}{lcc}
\hline Wedge interposition & Total number $(n)$ & Relative number (\%) \\
\hline Autologous spongiosa & 25 & 40 \\
ChroNos ${ }^{\circledR}$ & 31 & 49 \\
None & 7 & 11 \\
\hline
\end{tabular}

Table 3. Details on the mean Outerbridge score of the medial and lateral femorotibial compartment.

\begin{tabular}{lcc}
\hline Area of cartilage defect & Mean Outerbridge score \pm SD & Range \\
\hline Lateral femoral condyle & $0.4 \pm 0.7$ & $0-3$ \\
Lateral tibia & $0.5 \pm 0.8$ & $0-3$ \\
Lateral compartment & $0.9 \pm 1.4$ & $0-6$ \\
Medial femoral condyle & $2.5 \pm 1.3$ & $0-4$ \\
Medial tibia & $2.4 \pm 1.4$ & $0-4$ \\
Medial compartment & $4.8 \pm 2.5$ & $0-8$ \\
\hline
\end{tabular}

arthroscopic surgery before the HTO procedure or showed to have a meniscal lesion requiring partial meniscectomy at the time of the HTO procedure (Table 4). Regarding the lateral meniscus, there was only one case with an injury of the lateral meniscus that was treated by partial meniscectomy at the time of the HTO procedure. Coronal stability of the knee was given in all 63 cases by both intact medial and lateral collateral ligaments. Only two patients revealed a remarkedly sagittal instability by a total rupture of the anterior cruciate ligament (ACL) that was addressed using an autologous graft (semitendinosus tendon) at the time of the HTO procedure. Another two patients underwent ACL reconstruction surgery using an autologous graft at least six months before the HTO procedure. Still, they showed sagittal stability at the time of the HTO procedure.

\section{Functional outcome and KOOS}

A significant improvement in all five KOOS subscale scores during the follow-up could be observed $(p<0.00)$. A bivariate correlation analysis revealed a significant negative correlation between the postoperative KOOS subscores and the total Outerbridge score of the lateral compartment. 
Table 4. Details on the meniscal and ligamentous integrity of the affected knee at the time of the HTO procedure.

\begin{tabular}{lcc}
\hline $\begin{array}{l}\text { Meniscal and ligamentous } \\
\text { integrity of the knee }\end{array}$ & $\begin{array}{c}\text { Total } \\
\text { number }(n)\end{array}$ & $\begin{array}{c}\text { Relative } \\
\text { number }(\%)\end{array}$ \\
\hline Medial compartment & 18 & 29 \\
$\quad$ Intact meniscus & 13 & 20 \\
$\quad$ Meniscal lesion & 32 & 51 \\
$\quad$ Intact meniscal stump (partially resected) & & \\
Intercondylar region & 59 & 94 \\
$\quad$ Intact ACL & 2 & 3 \\
Ruptured ACL & 2 & 3 \\
ACL-graft & 63 & 100 \\
Intact PCL & 0 & 0 \\
Ruptured PCL & 0 & 0 \\
$\quad$ PCL-graft & & \\
Lateral compartment & 62 & 98 \\
Intact meniscus & 1 & 2 \\
Meniscal lesion & 0 & 0 \\
Intact meniscal stump (partially resected) & 0 \\
\hline
\end{tabular}

However, this did not apply to the KOOS Symptoms subscale. There were significantly lower (i.e., worse) KOOS subscales scores for patients with lateral cartilage defects (group B) compared to patients with no cartilage defects of the lateral compartment (group A) (Figure 1). From the preoperative to the postoperative visit, group A showed a significant increase in five KOOS subscale scores, whereas for group B a significant increase could be observed only for the KOOS-ADL (Activity of daily life) and KOOS-Pain score (Table 5). Regarding the preoperative KOOS subscores, no significant differences could be observed between both groups (Table 6). No statistically significant difference for both groups could be shown at the preoperative visit regarding the total KOOS score. However, at the postoperative visit, values of the total KOOS score were significantly higher for group A (Table 6).

\section{Pre- and postoperative alignment}

This study cohort's mean preoperative mechanical femorotibial angle was $172.9^{\circ} \pm 2.7^{\circ}$ of varus angulation. After the HTO procedure, the postoperative mean femorotibial angle was $181.4^{\circ} \pm 2.6^{\circ}$ of valgus. Preoperatively planned and postoperatively achieved femorotibial valgus angle did not differ significantly $\left(182.0^{\circ} \pm 1.1^{\circ}\right.$ vs. $\left.181.4^{\circ} \pm 2.6^{\circ}, p=0.1\right)$. The postoperative Mikulicz-line passed the tibial plateau at a mean width of $54.7 \% \pm 12.2 \%$, defined as the target area defined by Fujisawa [20]. However, a bivariate correlation analysis showed no significant correlation between the postoperative KOOS subscores and the postoperative femorotibial valgus angle $(r=-0.1$ to $0.60, p=0.4-0.8)$. The same applied to the tibial width of the postoperatively crossing Mikulicz-line: no correlation could be found with the postoperative KOOS subscores $(r=0.1, p=0.4-0.7)$.

\section{Complications}

Overall, complications were rare. There were three cases of which complications were documented. Two patients suffered from postoperative wound infections that could be successfully addressed solely by the prolonged administration of antibiotics without the need for operative revision surgery. In one case, a prolonged bone healing process was addressed properly by a prolonged period of partial weight-bearing. No case of a recurring postoperative varus alignment of the treated extremity has been documented. In two cases, conversion to TKA has been documented, the first after 6.2 years and the second after 8.5 years after the index procedure.

\section{Discussion}

Recent research has shown that the HTO procedure can significantly reduce pain and improve knee function in patients with medial osteoarthritis by delaying the progress of degeneration of the medial compartment [4]. The HTO procedure leads to unloading of the medial compartment by shifting the weightbearing axis towards a more lateralized one. By a four-degree mechanical femorotibial valgus angle, the load-bearing is assumed to be distributed equally between the medial and lateral compartment [21]. As such, great consensus exists between orthopaedic surgeons to aim for a slightly postoperative valgus alignment of the knee. Many authors suggest a postoperative femorotibial valgus angle of $5-10^{\circ}$ which equates the intersection of the weight-bearing line at $62-66 \%$ of the tibial width [21-25]. The ideal correction angle is believed to allow for sufficient unloading and regeneration of the medial compartment while at the same time avoiding overloading and degeneration of the lateral compartment [26-28].

Generally, the influence of the HTO procedure on laterally located cartilage defects of the knee remains controversial [29-31]. While a high-grade medial cartilage degeneration is generally believed to negatively impact the outcome, little is known about the lateral compartment's natural course of cartilage defects when performing HTO [32, 33]. For this reason, a special interest of this study focused on whether patients with asymptomatic mild to moderate cartilage defects of the lateral compartment are likewise expected to benefit from HTO. As such, the aim was to investigate whether these patients can achieve comparable results to patients with normal lateral knee compartments and thus can be regarded as equally indicated for HTO. Some studies suggest that asymptomatic mild to moderate focal cartilage defects of the lateral compartment can be very well accepted without negatively influencing the total benefit of the HTO procedure [34, 35].

However, from the data of the present study, a conclusion contradictory to this assumption can be drawn. Deducing from the results of this retrospective analysis, mild to moderate cartilage degeneration of the lateral compartment leads to significantly worse postoperative outcome scores than those with intact lateral knee compartments. This major finding is partially in concordance with a recent study from Hohloch et al. showing that cartilage defects of the lateral compartment are generally associated with lower outcome scores. However, statistical significance for this finding was lacking [29]. A recent study from Jin et al. concluded that grade $\geq 2$ cartilage defects of the lateral compartment feature a significant risk factor for failure of the HTO procedure [36]. 

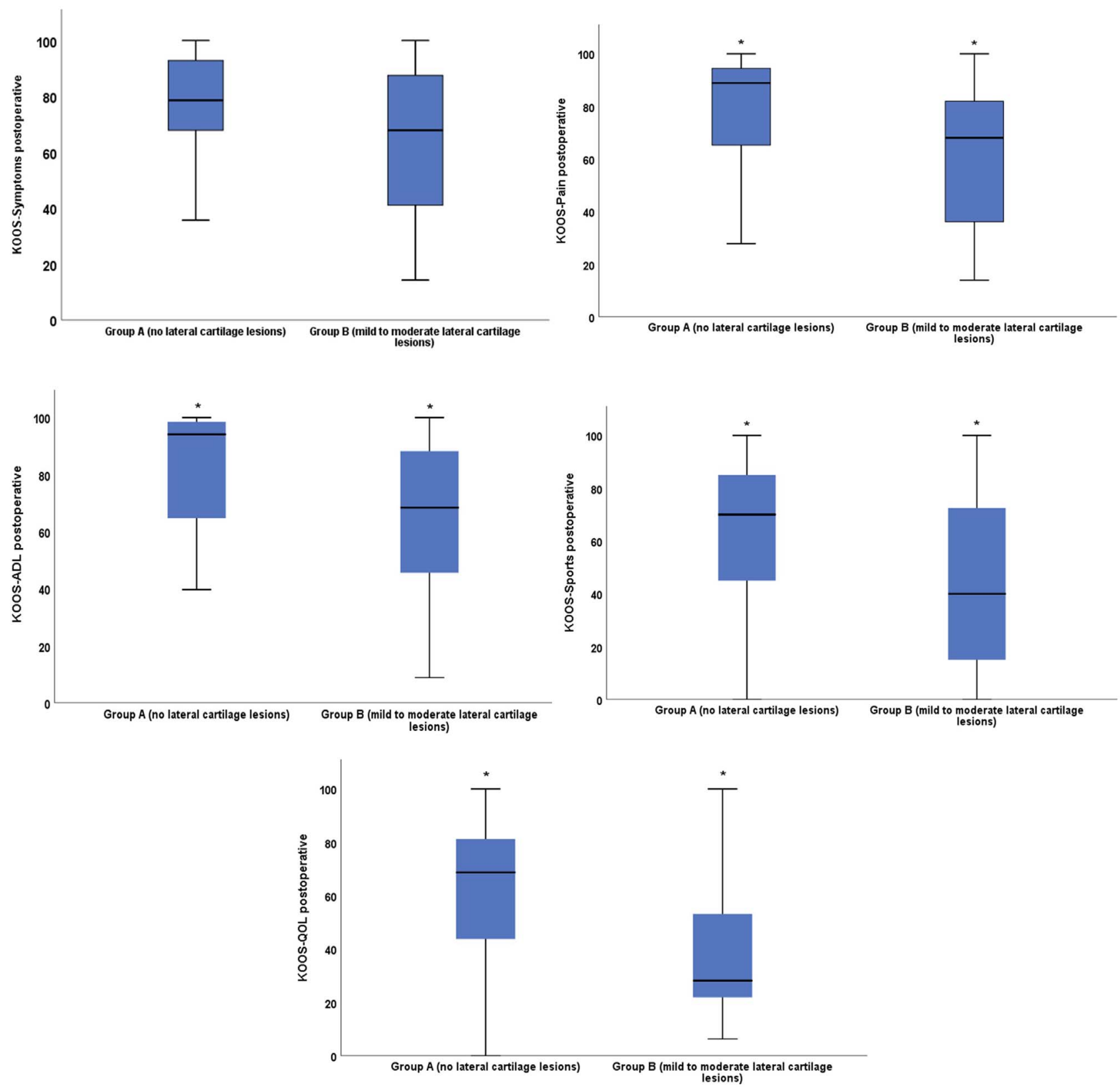

Figure 1. KOOS subscores at the postoperative visit for Group A and Group B.

Table 5. Changes of the KOOS subscores from the pre- to the postoperative visit for Group A and B.

\begin{tabular}{lcccc}
\hline & Group A (no lateral cartilage lesions, $n=39)$ & \multicolumn{2}{c}{ Group B (mild to moderate cartilage lesions, $n=24)$} \\
\hline $\begin{array}{l}\text { KOOS } \\
\text { subscore }\end{array}$ & $\begin{array}{c}\text { Mean increase from pre- to } \\
\text { postoperative visit }( \pm \text { SD) }\end{array}$ & $\begin{array}{c}P \text {-value for statistical } \\
\text { significance }\end{array}$ & $\begin{array}{c}\text { Mean increase from pre- to } \\
\text { postoperative visit }( \pm \text { SD) }\end{array}$ & $\begin{array}{c}P \text {-value for statistical } \\
\text { significance }\end{array}$ \\
\hline KOOS-Symptoms & $12.6 \pm 24.2$ & $0.00^{*}$ & $9.8 \pm 22.2$ & 0.10 \\
KOOS-Pain & $24.6 \pm 25.7$ & $0.00^{*}$ & $20.5 \pm 34.0$ & $0.03^{*}$ \\
KOOS-ADL & $21.8 \pm 24.1$ & $0.00^{*}$ & $19.5 \pm 34.2$ & $0.04^{*}$ \\
KOOS-Sports & $20.3 \pm 33.5$ & $0.00^{*}$ & $10.3 \pm 38.8$ & 0.31 \\
KOOS-QOL & $25.8 \pm 25.4$ & $0.00^{*}$ & $12.5 \pm 30.4$ & 0.12 \\
\hline
\end{tabular}

Significant differences are highlighted by an asterisk. SD: Standard deviation. 
Table 6. Mean pre- and postoperative KOOS subscores and statistical differences for Group A and Group B.

\begin{tabular}{lccc}
\hline KOOS subscore & $\begin{array}{c}\text { Mean KOOS score }( \pm \mathrm{SD}), \\
\text { Group A (no lateral } \\
\text { cartilage lesions) }\end{array}$ & $\begin{array}{c}\text { Mean KOOS score }( \pm \text { SD), } \\
\text { Group B (mild to moderate } \\
\text { cartilage lesions) }\end{array}$ & $\begin{array}{c}P \text {-value for } \\
\text { statistical } \\
\text { difference }\end{array}$ \\
\hline Preoperative & $62.8 \pm 27.9$ & $55.2 \pm 28.3$ & 0.42 \\
KOOS-Symptoms & $53.6 \pm 27.8$ & $42.9 \pm 26.6$ & 0.26 \\
KOOS-Pain & $60.2 \pm 32.4$ & $48.9 \pm 29.7$ & 0.29 \\
KOOS-ADL & $41.0 \pm 36.5$ & $36.5 \pm 41.6$ & 0.73 \\
KOOS-Sports & $31.3 \pm 27.8$ & $26.9 \pm 25.9$ & 0.64 \\
KOOS-QOL & $51.7 \pm 28.7$ & $39.5 \pm 25.1$ & 0.17 \\
KOOS-Total & & & $0.03^{*}$ \\
Postoperative & $78.3 \pm 17.4$ & $62.6 \pm 27.4$ & $0.00^{*}$ \\
KOOS-Symptoms & $81.1 \pm 19.1$ & $58.1 \pm 28.4$ & $0.01^{*}$ \\
KOOS-Pain & $83.8 \pm 19.4$ & $62.9 \pm 27.1$ & $0.02^{*}$ \\
KOOS-ADL & $65.2 \pm 29.4$ & $41.2 \pm 32.3$ & $0.01^{*}$ \\
KOOS-Sports & $59.3 \pm 25.6$ & $34.6 \pm 25.3$ & $0.01^{*}$ \\
KOOS-QOL & $73.8 \pm 20.3$ & $54.1 \pm 24.2$ & \\
KOOS-Total & & & \\
\hline
\end{tabular}

Statistically significant values are marked by an asterisk. SD: standard deviation.

These findings seem to be reasonable to some point: With increasing load-bearing of the lateral compartment, prior asymptomatic cartilage lesions likely tend to get symptomatic and may even tend towards progression [37]. From the data of this study, it can be hypothesized that even a meniscus intact lateral knee compartment at the time of surgery seems to be unable to fully compensate for the increased weight-bearing of the cartilage defects and thus not preventing them from getting symptomatic. This may be unproblematic if the cartilage of the lateral compartment shows normal integrity. An animalbased study of Ziegler et al. could demonstrate that neither macroscopic nor microscopic changes of the lateral tibiofemoral compartment can be found at six months after HTO [31]. However, it is stated that at least some progressive changes of the lateral meniscus after the HTO procedure can be observed [30, 38].

Moreover, concerns and criticism regarding the routinely performed arthroscopy before the HTO procedure have been raised recently, as some studies failed to show any relevance of the arthroscopic findings with the total outcome of the HTO procedure [39, 40]. Taking the present study's findings into account, the principle of a routinely performed arthroscopy prior to an osteotomy around the knee can be strongly encouraged. The presence of any focal lateral cartilage defects should then lead to a thorough re-evaluation of the planned osteotomy based on the findings of this study. Regarding minor findings of this study, the authors could demonstrate a negative bivariate correlation between the postoperative KOOS outcome score and the total degree of lateral cartilage degeneration according to the Outerbridge classification system. This suggests that a worse outcome with HTO can be expected with a higher degree of focal lateral cartilage defects. The postoperative valgus angle did not reveal any correlation with the final functional outcome scores, which concordance with existing literature [29, 41].

We are aware that the current study has several limitations. As this research was designed as a retrospective analysis, the level of evidence should generally be considered inferior to prospective cohort studies, and results may be biased. There was a statistically significant difference in the KellgrenLawrence Score of the medial compartment between both groups before surgery which might serve as a resource for some bias. However, this difference may be elicited by the relatively poor intra- and interrater variability of the Kellgren-LawrenceScore $[42,43]$. Especially the scoring between the second and third grade of the Kellgren-Lawrence-Score seems to warrant difficulties by the insufficient interrater variability in assessing the joint space narrowing on plain radiographs [14]. Yet the study's patient number and follow-up period seemed to be adequate despite a relatively large time span of the follow-up data acquisition [29, 41]. A further limitation is the availability of only one outcome score that was obtained pre-and postoperatively. Nonetheless, the KOOS is a powerful and validated tool that has also been validated for measurements of therapeutic effects of knee-related conditions [18].

\section{Conflict of interest}

The authors declare that they have no relevant financial or non-financial interests to report.

\section{Funding}

This research did not receive any specific funding.

\section{Ethical approval}

The ethics committee of the University of Wuerzburg has approved the retrospective study of this article. Written informed consent for participation was obtained from every individual.

\section{Informed consent}

Written and informed consent was obtained from every individual. 


\section{Authors contributions}

T. Heinz: Writing, Conceptualization, Drafting, Statistical analysis. S. Reppenhagen: Writing, Conceptualization, Methodology. M. Wagenbrenner: Statistical analysis, Writing, Visualization, Reviewing. K. Horas: Writing, Drafting, Investigation. M. Ohlmeier: Writing, Editing, Investigation, statistical analysis. T. Schäfer: Writing, Drafting, Editing, Formatting. M. Rudert: Writing, Reviewing, Supervision, Editing. T. Barthel: Writing, Methodology, Reviewing, Visualization. M. Weißenberger: Supervision, Writing, Reviewing, Editing, Conceptualization, Validation.

Acknowledgements. Not applicable.

\section{References}

1. Judith Fuchs RK, Scheidt-Nav C (2017) 12-month prevalence of osteoarthritis in Germany. J Health Monit 2(3), 51-55.

2. Sharma L, Chmiel JS, Almagor O, Felson D, Guermazi A, Roemer F, Lewis CE, Segal N, Torner J, Cooke TD, Hietpas J, Lynch J, Nevitt M (2013) The role of varus and valgus alignment in the initial development of knee cartilage damage by MRI: The MOST study. Ann Rheum Dis 72(2), 235-240.

3. Wu DD, Burr DB, Boyd RD, Radin EL (1990) Bone and cartilage changes following experimental varus or valgus tibial angulation. J Orthop Res 8(4), 572-585.

4. Brouwer GM, Tol AWV, Bergink AP, Belo JN, Bernsen RMD, Reijman M, Pols HAP, Bierma-Zeinstra SMA (2007) Association between valgus and varus alignment and the development and progression of radiographic osteoarthritis of the knee. Arthr Rheum 56(4), 1204-1211.

5. Andriacchi TP (1994) Dynamics of knee malalignment. Orthop Clin North Am 25(3), 395-403.

6. Vincent KR, Conrad BP, Fregly BJ (2012) The pathophysiology of osteoarthritis: A mechanical perspective on the knee joint. PM R 4, S3-S9.

7. Astephen Wilson JL, Deluzio KJ, Dunbar MJ (2011) The association between knee joint biomechanics and neuromuscular control and moderate knee osteoarthritis radiographic and pain severity. Osteoarthr Cartil 19, 186-193.

8. Jackson BD, Wluka AE, Teichtahl AJ (2004) Reviewing knee osteoarthritis - a biomechanical perspective. J Sci Med Sport 7, 347-357.

9. Jackson BD, Teichtahl AJ, Morris ME (2004) The effect of the knee adduction moment on tibial cartilage volume and bone size in healthy women. Rheumatology (Oxford) 43, 311-314.

10. Heller MO, Matziolis G, König C, Taylor WR, Hinterwimmer S, Graichen H, Hege HC, Bergmann G, Perka C, Duda GN (2007) Muskuloskelettale Biomechanik des Kniegelenks. Orthopäde 36(7), 628-634.

11. Sharma L, Song J, Felson DT, Cahue S, Shamiyeh E, Dunlop DD (2001) The role of knee alignment in disease progression and functional decline in knee osteoarthritis. JAMA 286(2), 188-195.

12. Santoso MB, Wu L (2017) Unicompartmental knee arthroplasty, is it superior to high tibial osteotomy in treating unicompartmental osteoarthritis? A meta-analysis and systemic review. J Orthop Surg Res 12(1), 50.
13. Dettoni F, Bonasia DE, Castoldi F, Bruzzone M, Blonna D, Rossi R (2010) High tibial osteotomy versus unicompartmental knee arthroplasty for medial compartment arthrosis of the knee: A review of the literature. Iowa Orthop J 30, 131-140.

14. Spahn G, Hofmann GO, von Engelhardt LV, Li M, Neubauer H, Klinger HM (2013) The impact of a high tibial valgus osteotomy and unicondylar medial arthroplasty on the treatment for knee osteoarthritis: A meta-analysis. Knee Surg Sports Traumatol Arthrosc 21(1), 96-112.

15. Brouwer RW, Huizinga MR, Duivenvoorden T, van Raaij TM, Verhagen AP, Bierma-Zeinstra SM, Verhaar JA (2014) Osteotomy for treating knee osteoarthritis. Cochrane Database Syst Rev (12), CD004019.

16. Dugdale TW, Noyes FR, Styer D (1992) Preoperative planning for high tibial osteotomy. The effect of lateral tibiofemoral separation and tibiofemoral length. Clin Orthop Relat Res (274), 248-264.

17. Roos EM, Lohmander LS (2003) The knee injury and osteoarthritis outcome score (KOOS): From joint injury to osteoarthritis. Health Qual Life Outcomes 1, 64.

18. Kessler S, Lang S, Puhl W, Stöve J (2003) The knee injury and osteoarthritis outcome score - a multifunctional questionnaire to measure outcome in knee arthroplasty. Z Orthop Ihre Grenzgeb 141(3), 277-282.

19. Paley D (2002) Realignment for mono-compartment osteoarthritis of the Knee. In: Principles of deformity correction, Vol. 1. Berlin Heidelberg New York, Springer-Verlag, pp. 479-485.

20. Fujisawa Y, Masuhara K, Shiomi S (1979) The effect of high tibial osteotomy on osteoarthritis of the knee. An arthroscopic study of 54 knee joints. Orthop Clin North Am 10(3), 585-608.

21. Mina C, Garrett WE Jr, Pietrobon R, Glisson R, Higgins L (2008) High tibial osteotomy for unloading osteochondral defects in the medial compartment of the knee. Am J Sports Med 36(5), 949-955.

22. Coventry MB (1979) Upper tibial osteotomy for gonarthrosis. The evolution of the operation in the last 18 years and long term results. Orthop Clin North Am 10(1), 191-210.

23. Coventry MB, Bowman PW (1982) Long-term results of upper tibial osteotomy for degenerative arthritis of the knee. Acta Orthop Belg 48(1), 139-156.

24. Coventry MB, Ilstrup DM, Wallrichs SL (1993) Proximal tibial osteotomy. A critical long-term study of eighty-seven cases. J Bone Joint Surg Am 75(2), 196-201.

25. Gao L, Madry H, Chugaev DV, Denti M, Frolov A, Burtsev M, Magnitskaya N, Mukhanov V, Neyret P, Solomin LN, Sorokin E, Staubli AE, Stone KR, Vilenskiy V, Zayats V, Pape D, Korolev A (2019) Advances in modern osteotomies around the knee. J Exp Orthop 6(1), 9.

26. Kanamiya T, Naito M, Hara M, Yoshimura I (2002) The influences of biomechanical factors on cartilage regeneration after high tibial osteotomy for knees with medial compartment osteoarthritis: Clinical and arthroscopic observations. Arthrosc J Arthrosc Relat Surg 18(7), 725-729.

27. Spahn G, Klinger HM, Harth P, Hofmann GO (2012) Cartilage regeneration after high tibial osteotomy. Results of an arthroscopic study. Z Orthop Unfall 150 (3), 272-279.

28. Odenbring S, Egund N, Lindstrand A, Lohmander LS, Willén H (1992) Cartilage regeneration after proximal tibial osteotomy for medial gonarthrosis. An arthroscopic, roentgenographic, and histologic study. Clin Orthop Relat Res 277, 210-216. 
29. Hohloch L, Kim S, Eberbach H, Izadpanah K, Mehl J, Niemeyer P, Südkamp NP, Bode G (2019) Improved clinical outcome after medial open-wedge osteotomy despite cartilage lesions in the lateral compartment PLoS One 14(10), e0224080.

30. Madry H, Ziegler R, Pape D, Cucchiarini M (2014) Structural changes in the lateral tibiofemoral compartment after high tibial osteotomy. Orthopade 43(11), 958-965.

31. Ziegler R, Goebel L, Cucchiarini M, Pape D, Madry H (2014) Effect of open wedge high tibial osteotomy on the lateral tibiofemoral compartment in sheep. Part II: Standard and overcorrection do not cause articular cartilage degeneration. Knee Surg Sports Traumatol Arthrosc 22(7), 1666-1677.

32. Floerkemeier S, Staubli AE, Schroeter S, Goldhahn S, Lobenhoffer P (2013) Outcome after high tibial open-wedge osteotomy: A retrospective evaluation of 533 patients. Knee Surg Sports Traumatol Arthrosc 21(1), 170-180.

33. Spahn G, Kirschbaum S, Kahl E (2006) Factors that influence high tibial osteotomy results in patients with medial gonarthritis: A score to predict the results. Osteoarthr Cartil 14(2), 190-195.

34. Moon H-S, Choi C-H, Jung M, Park S-H, Lee D-Y, Shin J-C, Kim S-H (2021) The effect of cartilage lesion in the lateral compartment of the knee on the surgical outcome of medial open-wedge high tibial osteotomy. J Knee Surg 34(5), 538-545.

35. Majima T, Yasuda K, Katsuragi R, Kaneda K (2000) Progression of joint arthrosis 10 to 15 years after high tibial osteotomy. Clin Orthop Relat Res (381), 177-184.

36. Jin C, Song E-K, Santoso A, Ingale PS, Choi I-S, Seon J-K (2020) Survival and risk factor analysis of medial open wedge high tibial osteotomy for unicompartment knee osteoarthritis. Arthrosc J Arthrosc Relat Surg 36(2), 535-543.
37. Felson DT, Niu J, Gross KD, Englund M, Sharma L, Cooke TDV, Guermazi A, Roemer FW, Segal N, Goggins JM, Lewis CE, Eaton C, Nevitt MC (2013) Valgus malalignment is a risk factor for lateral knee osteoarthritis incidence and progression: Findings from the Multicenter Osteoarthritis Study and the Osteoarthritis Initiative. Arthritis Rheum 65(2), 355-362.

38. Bick F, Iffland Y, Zimmermann E, Welsch F, Hoffmann R, Stein T (2019) The medial open-wegde osteotomy generates progressive intrameniscal integrity changes in the lateral knee compartment: A prospective MR-assessment after valgic osteotomy in the varus gonarthritic knee. Knee Surg Sports Traumatol Arthrosc 27(4), 1339-1346.

39. Keene JS, Dyreby JR, Jr (1983) High tibial osteotomy in the treatment of osteoarthritis of the knee. The role of preoperative arthroscopy. JBJS, 65(1), 36-42.

40. Keene JS, Monson DK, Roberts JM, Dyreby JR Jr (1989) Evaluation of patients for high tibial osteotomy. Clin Orthop Relat Res 243, 157-165.

41. 9Niemeyer P, Schmal H, Hauschild O, von Heyden J, Südkamp NP, Köstler W (2010) Open-wedge osteotomy using an internal plate fixator in patients with medial-compartment gonarthritis and varus malalignment: 3-year results with regard to preoperative arthroscopic and radiographic findings. Arthrosc J Arthrosc Relat Surg 26(12), 1607-1616.

42. Kohn MD, Sassoon AA, Fernando ND (2016) Classifications in brief: Kellgren-Lawrence classification of osteoarthritis. Clin Orthop Relat Res 474(8), 1886-1893.

43. Köse Ö, Acar B, Çay F, Yilmaz B, Güler F, Yüksel HY (2018) Inter- and Intraobserver Reliabilities of Four Different Radiographic Grading Scales of Osteoarthritis of the Knee Joint. J Knee Surg, 31(3), 247-253.

Cite this article as: Heinz T, Reppenhagen S, Wagenbrenner M, Horas K, Ohlmeier M, Schäfer T, Rudert M, Barthel T \& Weißenberger M (2021) Focal cartilage defects of the lateral compartment do influence the outcome after high tibial valgus osteotomy. SICOT-J 7, 44 